\title{
Vasoactive Agents for Hepatorenal Syndrome: A Mixed Treatment Comparison Network Meta-Analysis and Trial Sequential Analysis of Randomized Clinical Trials
}

\author{
Kannan Sridharan, MBBS, MD, DM ${ }^{7}$ and Gowri Sivaramakrishnan, $M^{2} S^{2}$ \\ 'School of Health Sciences, College of Medicine, Nursing and Health Sciences, Fiji National University, Suva, Fiji; ${ }^{2}$ School of Oral Health, College of \\ Medicine, Nursing and Health Sciences, Fiji National University, Suva, Fiji.
}

\begin{abstract}
BACKGROUND: Hepatorenal syndrome (HRS) is a common complication among patients with cirrhosis, primarily attributable to vasodilation of renal vessels. Vasoactive agents are commonly used to treat HRS. The present network meta-analysis compares the vasoactive agents used in HRS.

METHODS: We searched electronic databases for appropriate randomized controlled clinical trials in patients with HRS, comparing active interventions with either placebo or standard of care. The primary outcome was complete HRS reversal; secondary outcomes included partial HRS reversal, mortality, adverse events, and cardiovascular adverse events. The data were pooled using a random effects model. We also carried out direct comparisons for the primary outcome with trial sequential analysis.
\end{abstract}

RESULTS: A total of 16 studies were included in the systematic review. Rates of complete HRS reversal were significantly higher with terlipressin and noradrenaline combined with albumin than with placebo (OR 6.65, 95\% CI: 2.08-21.31 and 6.81, 95\% CI: 1.87-24.83, respectively). No significant differences were observed in terms of mortality, partial HRS reversal, or adverse events for any of the interventions. However, cardiovascular adverse events were significantly higher with continuous-infusion terlipressin/albumin (OR 7.07, 95\% CI: 1.23-40.62), bolus terlipressin/albumin (OR 7.39, 95\% CI: 1.89, 28.94), octreotide/midodrine/albumin (OR 9.85, 95\% CI: 1.1, 88.1), and noradrenaline/albumin (OR 15.24, 95\% CI: $2.1,112.6)$ than with albumin alone. Trial sequential analyses revealed adequate evidence to conclude that terlipressin combined with albumin was effective in achieving complete HRS reversal.

DISCUSSION: Terlipressin combined with albumin shows strong evidence of improving short-term survival in patients with type 1 but not type 2 HRS. Through indirect comparison, noradrenaline with albumin was also associated with significant benefits in terms of HRS reversal.

Protocol registration: This systematic review protocol is registered with PROSPERO, registration number CRD42017069818.

Electronic supplementary material The online version of this article (https://doi.org/10.1007/s11606-017-4178-8) contains supplementary material, which is available to authorized users.

Received July 3, 2017

Revised July 19, 2017

Accepted August 28, 2017

Published online September 18, 2017
KEY WORDS: terlipressin; noradrenaline; octreotide; cirrhosis; midodrine. J Gen Intern Med 33(1):97-102

DOI: $10.1007 / \mathrm{s} 11606-017-4178-8$

(C) Society of General Internal Medicine 2017

\section{INTRODUCTION}

Hepatorenal syndrome (HRS) is characterized by reduced renal function due to diminished blood flow to the kidneys in advanced stages of cirrhosis. Among patients with cirrhosis, the prevalence of HRS ranges from 4 to $39 \%,{ }^{1}$ and is frequently precipitated by spontaneous bacterial peritonitis. ${ }^{2}$ Mortality

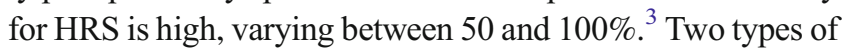
HRS have been identified: type 1 is associated with rapid and progressive impairment of renal function, with elevated serum creatinine within 2 weeks, while type 2 HRS has a slower time course. $^{4}$

Guidelines recommend the use of vasoconstrictors such as vasopressin analogs, noradrenaline, octreotide, and midodrine with albumin as first-line treatment in the management of HRS. $^{5,6}$ Direct pairwise meta-analyses have shown that the vasoconstrictors noradrenaline and terlipressin may both be useful in reversing HRS. ${ }^{7,8}$ The efficacy of renal vasodilators such as low-dose dopamine have also been explored in HRS. ${ }^{9}$ However, not all vasoactive agents have been assessed in direct comparison trials. Mixed treatment network metaanalysis compares the interventions available for treating a condition through a common comparator, and thus may be helpful for comparing the relative effects of the interventions. ${ }^{10}$ The purpose of our study was to conduct a network meta-analysis to compare the interventions used for treating HRS, with complete HRS reversal as the primary outcome measure.

\section{METHODS}

We registered the protocol for this review in the PROSPERO database (CRD42017069818). We searched PubMed and the Cochrane Central Register of Controlled Trials (CENTRAL) using an online strategy (Supplementary Appendix 1). No limits were applied for either publication year or language, and the search was completed on 20 June 2017 . We manually 
searched the references of retrieved articles for additional references.

We included randomized controlled clinical trials conducted among adults with either type 1 or type 2 HRS, where one active medication was compared with either placebo or another active medication. Complete HRS reversal was the primary outcome measure, and secondary outcome measures included partial reversal of HRS, mortality, adverse events, and cardiovascular adverse events.

Two authors independently performed the literature search and extracted information regarding the trial site, year, trial methods, participants, interventions, and outcomes; disagreements were resolved through discussion. Our meta-analysis was carried out according to the Preferred Reporting Items for Systematic Reviews and Meta-Analyses (PRISMA) guidelines. ${ }^{11}$ We assessed the risk of bias of included studies using the Cochrane risk of bias tool, ${ }^{12}$ and publication bias using funnel plots as well as the Egger regression test. ${ }^{13}$ A random effects model was used to assess the direct and mixed treatment effect estimates. Odds ratios (95\% confidence interval) were used as the effect estimate for all outcomes. We assessed for inconsistency between direct and indirect pooled effect estimates through the use of $\overline{\mathrm{H}}$ statistics, wherein a value of $<3$ was considered minimal, 3-6 modest, and $>6$ large. ${ }^{14}$ Subgroup analyses were carried out for type 1 and 2 HRS for complete HRS reversal and mortality. Trial sequential analysis (TSA; Copenhagen Trial Unit, Copenhagen, Denmark) was performed to assess the likelihood of the occurrence of either a type I or type II error in comparisons of terlipressin combined with albumin and noradrenaline combined with albumin compared to albumin alone, according to the information size achieved until the date of complete HRS reversal and mortality. ${ }^{15}$ We used the O'Brien-Fleming alphaspending function method to assess the statistical significance of the pooled estimate. ${ }^{16}$ MetaXL was used for the analyses of pooled estimates. ${ }^{17}$ Direct, indirect, and mixed treatment comparison estimates were generated for all eligible outcomes, and a network meta-analysis was carried out for the mixed treatment comparison estimates. Grading of the evidence for key comparisons was carried out using the Grading of Recommendations Assessment, Development and Evaluation (GRADE) working group approach. ${ }^{12}$

\section{RESULTS}

A total of 232 articles were identified, among which $16^{18-33}$ studies were included in the systematic review and metaanalysis (Supplementary Appendix Fig. 1). Key characteristics of the included studies are provided in Supplementary Appendix Table 1. Five studies compared the combination of noradrenaline and albumin with terlipressin and albumin; six compared the combination of terlipressin and albumin with albumin alone; one each compared 1) combined terlipressin and albumin with combined midodrine, octreotide, and albumin; 2) octreotide with albumin; 3) combined terlipressin and albumin with combined dopamine, furosemide, and albumin; and 4) continuous infusion of terlipressin with albumin. One study compared combined noradrenaline and albumin with midodrine, octreotide, and albumin. Two of the included studies $^{23,33}$ were conference abstracts, for which risk of bias for the individual domains could not be assessed. The risk of bias for the individual domains for the included studies is presented in Supplementary Appendix Figure 2.

\section{Pooled Results}

Primary Outcome. A total of 12 studies including 762 participants were pooled for the network meta-analysis of complete reversal. Five studies compared the combination of noradrenaline/albumin with terlipressin/albumin, and four compared terlipressin/albumin with albumin. One study each compared 1) terlipressin/albumin with combined midodrine/octreotide/albumin, 2) noradrenaline/albumin with midodrine/octreotide/albumin, and 3) continuous infusion of terlipressin/albumin with terlipressin bolus/albumin.

Of the 12 studies that investigated complete HRS reversal, the average duration of the interventions was 2 weeks. Seven studies assessed outcomes at 3 months, and one each assessed outcomes at 15 days, 30 days, 6 months, and until death or liver transplantation. The sample size ranged from 22 to 196 participants. Patient age ranged from 48.2 to 65 years, with women comprising 13 to $46.4 \%$ of participants. In all three studies comparing two vasoactive agents, interventions were administered for 2 weeks, with follow-up of 3 months. Sample size ranged from 23 to 71 participants, aged 52.9 to 65 years, with women comprising 32.3 to $34.9 \%$.

Mixed treatment comparisons of the interventions with albumin showed a significant increase in the proportion of individuals with complete HRS reversal with terlipressin/ albumin (OR: 6.7, 95\% CI: 2.1-21.3, Fig. 1), noradrenaline/ albumin (OR 6.8, 95\% CI: 1.9-24.8), and continuous infusion of terlipressin/albumin (OR: 9.9, $95 \mathrm{CI}: 2.2,44.2)$, but not with midodrine/octreotide/albumin (OR 0.3, 95\% CI: 0.02, 3.1). Small inconsistencies were observed between the direct and indirect comparison pooled estimates ( $\overline{\mathrm{H}}$ ranging from 1 to 1.8).

Secondary Outcomes. No significant differences were observed between the interventions in the mixed treatment estimates for partial HRS reversal (seven studies, 395 participants; Table 1), HRS recurrence (four studies, 92 participants), mortality (15 studies, 908 participants, as follows: seven studies assessing mortality at 3 months, two each at 15 and 30 days, one at 6 months, one with follow-up until death or liver transplantation, and two studies that were conference abstracts with no mention of follow-up periods) or adverse events (eight studies, 630 participants). Data from a total of seven studies including 377 participants were pooled for the analysis of differences in 


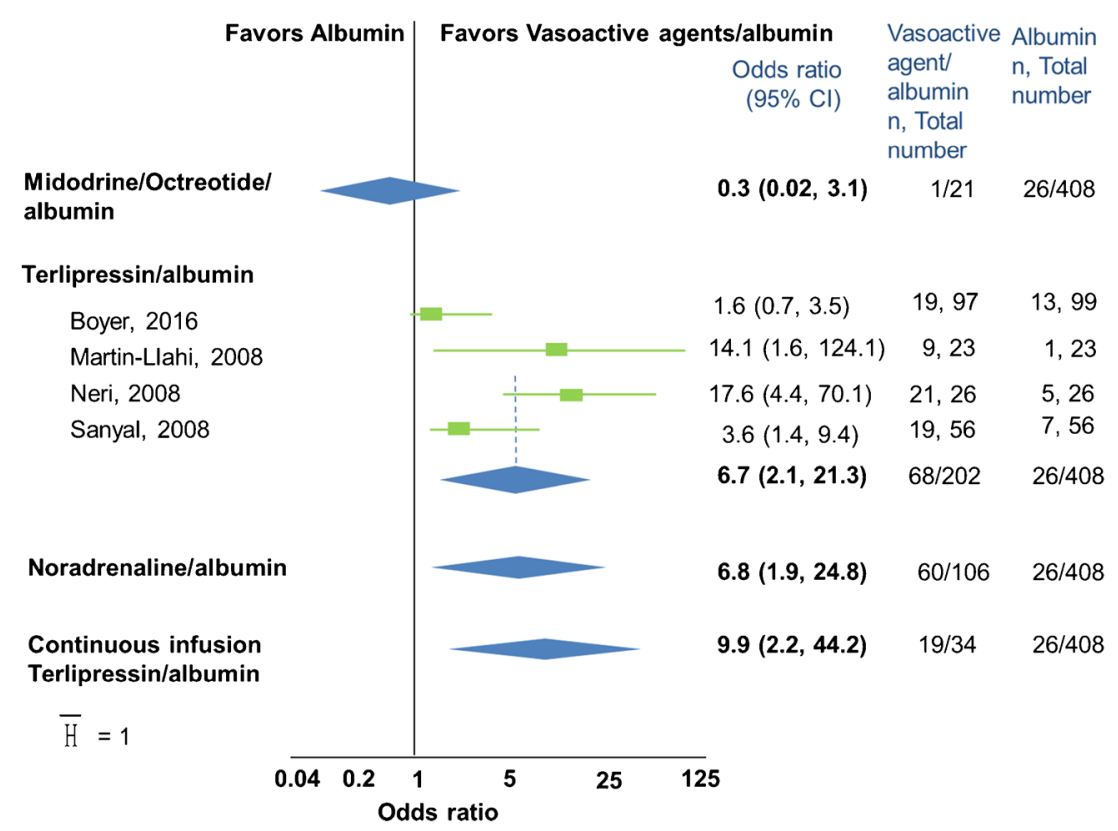

Figure 1 Forest plot of the mixed treatment comparison (MTC) estimates for complete HRS reversal. Terlipressin, noradrenaline, and continuous-infusion terlipressin combined with albumin demonstrated significantly improved complete HRS reversal over albumin alone.

cardiovascular adverse events, and all except the combination of dopamine, furosemide, and albumin were associated with high risk (Table 1).

\section{Subgroup Analyses}

Subgroup analyses for complete HRS reversal in type 1 HRS patients revealed a benefit for terlipressin/albumin (OR 5.8, 95\% CI: 1.5-22.1; Fig. 2), noradrenaline/albumin (OR 6.4, 95\% CI: 1.5-28), and continuous-infusion terlipressin/albumin (OR 8.7, 95\% CI: 1.7-44.1) compared to albumin alone. No significant differences were observed for terlipressin/albumin (OR 1.23, 95\% CI: $0.1-4.2$ ) or midodrine/octreotide/albumin (OR 5, 95\% CI: 0.2-146.4) for type 2 HRS. Similarly, no significant differences were observed in mortality risk for type 1 or type 2 HRS.

\section{Sensitivity Analyses}

No publication bias was detected using either funnel plots or statistical tests for the comparison of combined noradrenaline and albumin with combined terlipressin and albumin, for either complete HRS reversal (Egger regression, $p=0.38$ ) or mortality $(p=0.7)$, or for the comparison of combined terlipressin and albumin with albumin alone for mortality ( $p=0.07)$. Due to the paucity of studies, publication bias could not be assessed for other comparisons.

The exclusion of studies reporting outcomes at other than 3 months produced no significant changes in the mixed treatment comparison pooled estimates. Similarly, exclusion of the results from conference abstracts resulted in no significant changes to our pooled estimates.

\section{Trial Sequential Analysis}

Trial sequential analysis suggested that only the HRS complete reversal comparison between terlipressin/albumin and albumin alone was robust against error (Fig. 3). The reported benefit for HRS reversal for noradrenaline/albumin compared with albumin alone could represent a type I error. The lack of mortality benefit for both terlipressin/albumin and noradrenaline/albumin compared to albumin alone is at risk of being a type II error.

\section{Grading the Evidence}

Our grading of the quality of evidence for key comparisons is provided in Table 2 . We conclude that the quality of evidence

Table 1 Mixed Treatment Comparison Pooled Estimates for the Secondary Outcomes

\begin{tabular}{|c|c|c|c|c|c|}
\hline Interventions & $\begin{array}{l}\text { Partial reversal } \\
(95 \% \text { CI })\end{array}$ & $\begin{array}{l}\text { Recurrence } \\
(95 \% \text { CI) }\end{array}$ & $\begin{array}{l}\text { Mortality } \\
(95 \% \text { CI) }\end{array}$ & $\begin{array}{l}\text { Adverse events } \\
\text { (95\% CI) }\end{array}$ & $\begin{array}{l}\text { Cardiovascular } \\
\text { adverse } \\
\text { events }(95 \% \text { CI })\end{array}$ \\
\hline Noradrenaline/albumin & $0.23(0.01-3.8)$ & $0.14(0-4.7)$ & $0.6(0.3-1.4)$ & $0.7(0.2-2.7)$ & $15.2(2.1-112.6)^{*}$ \\
\hline Terlipressin/albumin & $0.78(0.18-3.5)$ & $0.3(0-6.2)$ & $0.6(0.4-1.1)$ & $1.9(0.7-5)$ & $7.4(1.9-28.9)^{*}$ \\
\hline Continuous-infusion terlipressin/albumin & $0.86(0.13-5.8)$ & Not available & $0.35(0.1-1.1)$ & $0.6(0.2-2.5)$ & $7.1(1.2-40.6)^{*}$ \\
\hline Midodrine/octreotide/albumin & $1.0(0.13-8.5)$ & $0.2(0-11.2)$ & $1.2(0.3-4.2)$ & $2.2(0.5-10.9)$ & $9.9(1.1-88.1)^{*}$ \\
\hline Dopamine/furosemide/albumin & Not available & Not available & $0.7(0.2-2.4)$ & $0.9(0.1-6.8)$ & $3.5(0.4-32.4)$ \\
\hline
\end{tabular}

*Indicates statistical significance 


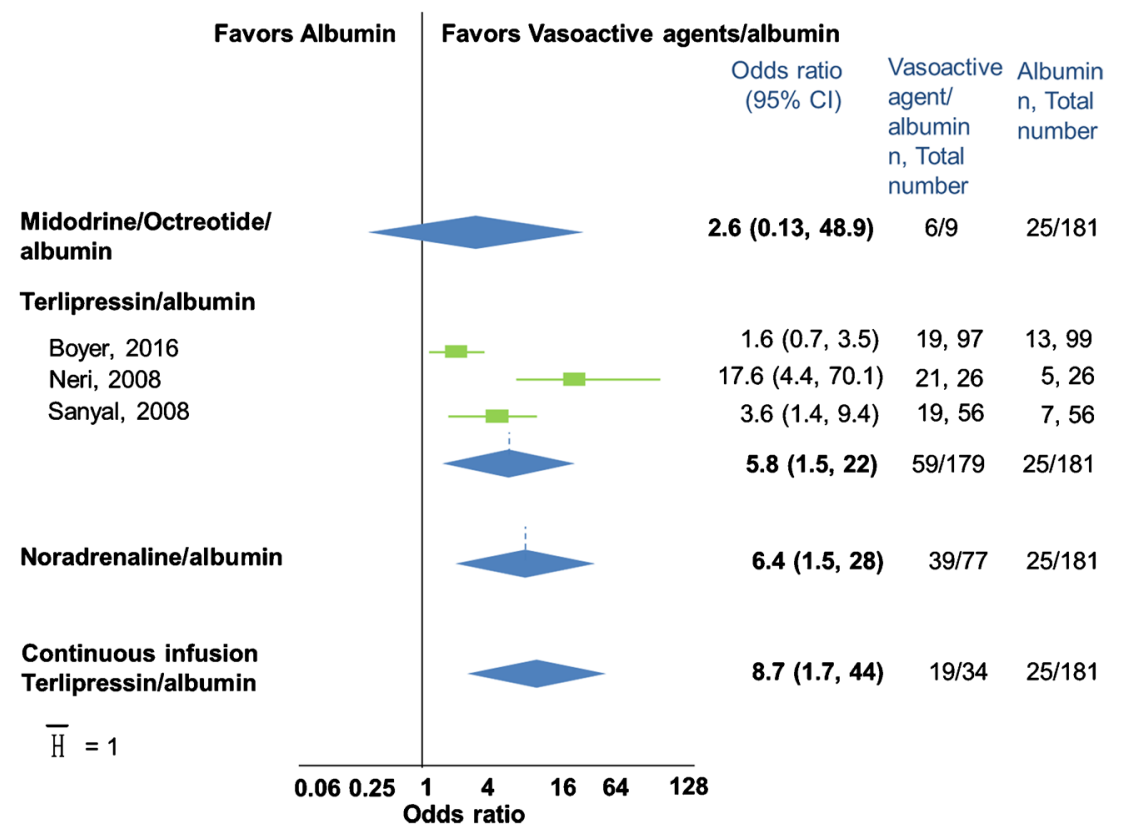

Figure 2 Forest plot of mixed treatment comparison (MTC) estimates for complete HRS reversal in type 1 HRS. Terlipressin, noradrenaline, and continuous-infusion terlipressin combined with albumin significantly improved HRS reversal over albumin alone in patients with type 1 HRS.

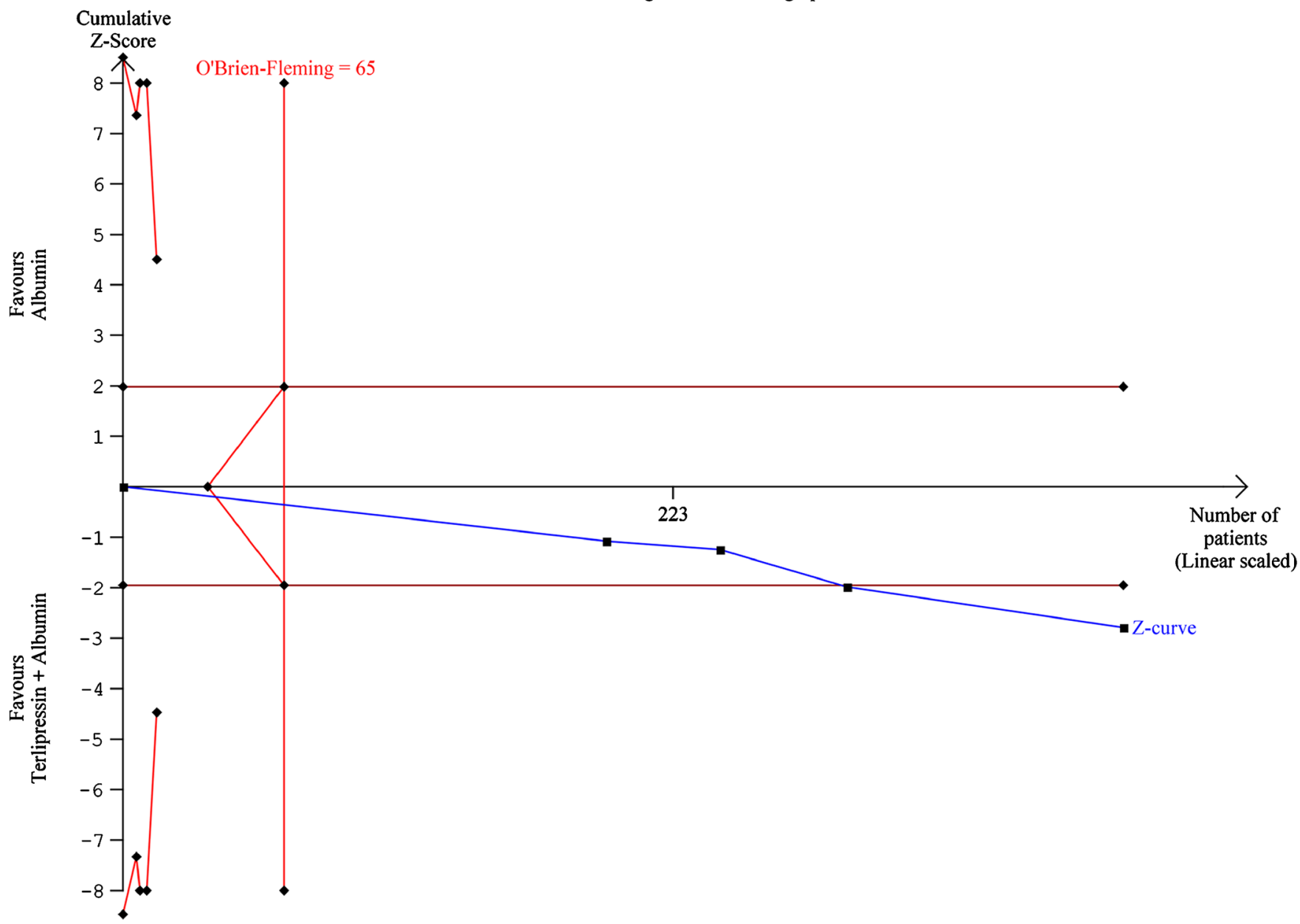

$\mathrm{O}^{\prime}$ Brien-Fleming is a Two-sided graph

Figure 3 Trial sequential analysis for the comparison of terlipressin/albumin with albumin for complete HRS reversal. The red horizontal lines indicate the conventional alpha boundaries, and the blue line depicts the pooled estimate of direct comparisons between terlipressin/albumin and albumin. 
Table 2 Grading the Quality of Evidence for Key Comparisons

\begin{tabular}{|c|c|c|c|c|}
\hline \multirow[t]{2}{*}{ Comparisons } & \multicolumn{2}{|c|}{$\begin{array}{l}\text { Illustrative comparative risks } \\
\text { (95\% confidence intervals) }\end{array}$} & \multirow{2}{*}{$\begin{array}{l}\text { Effect estimate and } \\
\text { quality of evidence } \\
\text { for direct comparisons }\end{array}$} & \multirow{2}{*}{$\begin{array}{l}\text { Effect estimate and } \\
\text { quality of evidence } \\
\text { or mixed treatment } \\
\text { comparisons }\end{array}$} \\
\hline & Assumed risk* & Corresponding risk & & \\
\hline $\begin{array}{l}\text { HRS complete reversal } \\
\text { or the combined terlipressin } \\
\text { and albumin with albumin }\end{array}$ & $123 / 1000$ & 489 (235-749) & $\begin{array}{l}6.7[2.1,21.3] \\
\oplus \Theta \Theta \ominus \\
\text { Very low } \dagger \ddagger 8\end{array}$ & $\begin{array}{l}6.7[2.1,21.3] \\
\oplus \Theta \Theta \ominus \\
\text { Very low } \dagger \ddagger \S\end{array}$ \\
\hline $\begin{array}{l}\text { HRS complete reversal for } \\
\text { the combined noradrenaline } \\
\text { and albumin with albumin }\end{array}$ & $123 / 1000$ & $492(212-782)$ & Not available & $\begin{array}{l}6.8[1.9,24.8] \\
\oplus \Theta \Theta \Theta \\
\text { Very low } \dagger \S \|\end{array}$ \\
\hline $\begin{array}{l}\text { HRS complete reversal for } \\
\text { the continuous infusion of } \\
\text { terlipressin compared to albumin }\end{array}$ & $123 / 1000$ & $580(246-859)$ & Not available & $\begin{array}{l}9.9[2.2,41.2] \\
\mathrm{ND}\end{array}$ \\
\hline
\end{tabular}

*Assumed risk was the median control group risk across studies

${ }^{\dagger}$ Downgraded one level for inclusion of studies with high risk of bias

${ }^{7}$ Downgraded one level because publication bias could not be ruled out

${ }^{s}$ Downgraded one level for serious limitations in the precision of estimates

"Downgraded one level for lack of direct estimate available and the presence of first-order loop in the mixed treatment comparison analysis

ND: Not determined-due to serious limitations in the precision of the estimates, publication bias could not be assessed and studies with high risk of bias were included for evaluating the outcomes

Very low quality: We are very uncertain about the estimate

is very low due to serious limitations with regard to risk of bias, publication bias, and precision of the estimates.

\section{DISCUSSION}

We conducted a network meta-analysis to compare the pharmacological treatment of HRS in patients with cirrhosis. Results revealed that there was adequate evidence that the combination of terlipressin and albumin was superior to albumin alone in achieving complete reversal of HRS in type 1 patients. We also found evidence that the combination of noradrenaline and albumin and the combination of continuous infusion of terlipressin and albumin were more effective than albumin alone for type 1 HRS, though our TSA analysis suggests that this is a preliminary finding, requiring additional evidence. The combination of midodrine/octreotide and albumin was not found to be superior to albumin alone. No differences were observed between the interventions in terms of mortality, adverse events, or HRS recurrence. Regimens that included terlipressin, noradrenaline, or the combination of midodrine/ octreotide were associated with a significantly greater risk of cardiovascular adverse events compared to albumin alone.

Vasodilation of the renal arteries is the underlying pathophysiology for HRS. ${ }^{34}$ The combination of vasoconstrictors and albumin is the first-line management for type $1 \mathrm{HRS}^{35}$ Profound splanchnic vasodilation in patients with portal hypertension also results in reduced renal perfusion. ${ }^{36}$ Low-dose dopamine selectively dilates renal blood vessels and can ameliorate the signs and symptoms of HRS. Our results suggest that terlipressin may perform better, likely because it promotes vasoconstriction in both systemic and splanchnic blood vessels, and also dilates intrahepatic portal vessels, reducing portal hypertension. ${ }^{37}$ A previous meta-analysis comparing terlipressin with noradrenaline concluded that noradrenaline was an attractive alternative to terlipressin. ${ }^{38}$ Although both performed well in terms of achieving complete reversal of type 1 HRS, none of the agents reduced mortality or HRS recurrence after cessation of vasoconstrictor administration, a finding similar to those of previous meta-analyses. ${ }^{7,8}$ Therefore, adding vasoconstrictors may temporarily improve the patient's symptoms, but subsequent liver transplantation may be necessary for long-term survival. ${ }^{39}$ One advantage with a TSA approach is that it allows the analyst to assess the likelihood of error, either reporting a difference that does not exist (type I) or reporting no difference when there is one (type II). Our finding that terlipressin and albumin were superior to albumin alone appears robust against error, though our other findings require additional trials to protect against error.

Our study has a number of limitations. First, we did not search Embase for potential articles, though our manual review of included studies revealed no additional references. Second, variations in vasoconstrictor dose were not assessed. Finally, the specific criteria for establishing HRS in patients were not provided in most studies.

We conclude that there is adequate evidence to recommend the combination of terlipressin and albumin for reversing type 1 HRS. Through indirect comparison, noradrenaline with albumin was also found to be associated with significant benefits in terms of HRS reversal; however, our TSA analysis suggests that the data are not adequate to exclude a type I error. Thus further studies are needed.

Prior Presentations: This work has not been presented previously.

Corresponding Author: Kannan Sridharan, MBBS, MD, DM; School of Health Sciences, College of Medicine, Nursing and Health SciencesFiji National University, Suva, Fiji (e-mail: skannandr@gmail.com).

Author Contributions KS had full access to all of the data in the study, and takes responsibility for the integrity of the data, the accuracy of the data analysis, and the final decision to submit for publication. Study concept and design: KS, GS. Acquisition, analysis, 
or interpretation of data: KS, GS. Drafting of the manuscript: KS, GS. Critical revision of the manuscript for important intellectual content: KS, GS,. Statistical analysis: KS, GS.

Funding No funding was obtained for this study.

Compliance with Ethical Standards:

Conflict of Interest: The authors declare that they do not have a conflict of interest.

\section{REFERENCES}

1. Ginès A, Escorsell A, Ginès $\mathbf{P}$, Saló $\mathbf{J}$, et al. Incidence, predictive factors, and prognosis of the hepatorenal syndrome in cirrhosis with ascites. Gastroenterol. 1993;105:229-36.

2. Ginès P, Martin PY, Niederberger M. Prognostic significance of renal dysfunction in cirrhosis. Kidney Int Suppl. 1997;61:S77-82.

3. Heidemann $\mathbf{J}$, Bartels $\mathbf{C}$, Berssenbrügge $\mathbf{C}$, Schmidt $\mathbf{H}$, Meister T. Hepatorenal Syndrome: Outcome of response to therapy and predictors of survival. Gastroenterol Res Pract. 2015:2015. https://doi.org/10. $1155 / 2015 / 457613$.

4. Salerno F, Gerbes A, Ginès $\mathbf{P}$, Wong F, Arroyo V. Diagnosis, prevention and treatment of hepatorenal syndrome in cirrhosis. Gut. 2007;56:13108.

5. European Association for the Study of the Liver. EASL clinical practice guidelines on the management of ascites, spontaneous bacterial peritonitis, and hepatorenal syndrome in cirrhosis. J Hepatol. 2010;53:397417.

6. Runyon BA. Management of adult patients with ascites due to cirrhosis: Update 2012. American Association for the Study of Liver Diseases. Available at: https://www.aasld.org/sites/default/files/guideline_documents/adultascitesenhanced.pdf (Accessed on 22 Jun 2017).

7. Fabrizi F, Dixit V, Martin P. Meta-analysis: terlipressin therapy for the hepatorenal syndrome. Aliment Pharmacol Ther. 2006;24:935-44.

8. Gluud LL, Christensen $\mathbf{K}$, Christensen E, Krag A. Terlipressin for hepatorenal syndrome. Cochrane Database Syst Rev. 2012;(9):CD005162. https://doi.org/10.1002/14651858.CD005162. pub3.

9. ASMKBYDSS A. Prospective randomized controlled trial of concurrent triple therapy for hepatorenal syndrome. J Gastroenterol Hepatol. 2004; 19(S7):A119.

10. Greco T, Biondi-Zoccai G, Saleh O, et al. The attractiveness of network meta-analysis: a comprehensive systematic and narrative review. Heart Lung Vessel. 2015;7:133-142.

11. Hutton B, Salanti G, Caldwell DM, et al. The PRISMA Extension Statement for Reporting of Systematic Reviews Incorporating Network Meta-analyses of Health Care Interventions: Checklist and Explanations. Ann Intern Med. 2015;162:777-784.

12. Higgins JPT, Green S, eds. Cochrane Handbook for Systematic Reviews of Interventions. Version 5.1.0. Available from www.cochrane-handbook. org (last accessed on 1 May 2017).

13. Sedgwick P, Marston L. How to read a funnel plot in a meta-analysis. BMJ. 2015;351:h4718.

14. Higgins JPT, Thompson SG. Quantifying heterogeneity in a metaanalysis. Stat Med. 2002;21:1539-1558.

15. Thorlund K, Engstrom J, Wetterslev J, Brok J, Imberger G, Gluud C. Trial sequential analysis. Copenhagen trial unit. Available at: http:// www.ctu.dk/tools-and-links/trial-sequential-analysis.aspx (Accessed on 22 June 2017).

16. O'Brien PC, Fleming TR. A multiple testing procedure for clinical trials. Biometrics. 1979;35:549-56.

17. Barendregt JJ, Doi SA. MetaXL user guide. Available at: http://www. epigear.com/index_files/MetaXL\%20User\%20Guide.pdf (Accessed on 21 July 2017).

18. Alessandria C, Ottobrelli A, Debernardi-Venon W, et al. Noradrenalin vs terlipressin in patients with hepatorenal syndrome: a prospective, randomized, unblinded, pilot study. J Hepatol. 2007 ; 47:499-505.

19. Boyer TD, Sanyal AJ, Wong F, REVERSE study investigators, et al. Terlipressin plus albumin is more effective than albumin alone in improving renal function in patients with cirrhosis and hepatorenal syndrome type 1. Gastroenterology. 2016;150:1579-1589.e2.
20. Cavallin M, Kamath PS, Merli M, Italian Association for the study of the liver study group on hepatorenal syndrome, et al. Terlipressin plus albumin versus midodrine and octreotide plus albumin in the treatment of hepatorenal syndrome: A randomized trial. Hepatology. 2015;62:56774 .

21. Cavallin M, Piano S, Romano A, et al. Terlipressin given by continuous intravenous infusion versus intravenous boluses in the treatment of hepatorenal syndrome: A randomized controlled study. Hepatology. 2016;63:983-92.

22. Ghosh S, Choudhary NS, Sharma AK, et al. Noradrenaline vs terlipressin in the treatment of type 2 hepatorenal syndrome: a randomized pilot study. Liver Int. 2013;33:1187-93.

23. Indrabi RA, Javid G, Zargar SA, et al. Noradrenaline is equally effective as terlipressin in reversal of type 1 hepatorenal syndrome: A randomized prospective study. J Clin Exp Hep. 2013;13:S97.

24. Martín-Llahi M, Pépin MN, Guevara M, Diaz F, Torre A, TAHRS Investigators, et al. Terlipressin and albumin vs albumin in patients with cirrhosis and hepatorenal syndrome: a randomized study. Gastroenterol. 2008; 134:1352-9.

25. Neri S, Pulvirenti D, Malaguarnera M, et al. Terlipressin and albumin in patients with cirrhosis and type I hepatorenal syndrome. Dig Dis Sci. 2008;53:830-5.

26. Pomier-Layrargues G, Paquin SC, Hassoun Z, Lafortune M, Tran A. Octreotide in hepatorenal syndrome: a randomized, double-blind, placebo-controlled, crossover study. Hepatology. 2003;38:238-43.

27. Sanyal AJ, Boyer T, Garcia-Tsao G, Terlipressin Study Group, et al. A randomized, prospective, double-blind, placebo-controlled trial of terlipressin for type 1 hepatorenal syndrome. Gastroenterol. 2008; 134:1360-8.

28. Sharma P, Kumar A, Shrama BC, Sarin SK. An open label, pilot, randomized controlled trial of noradrenaline versus terlipressin in the treatment of type 1 hepatorenal syndrome and predictors of response. Am J Gastroenterol. 2008;103:1689-97.

29. Singh V, Ghosh S, Singh B, et al. Noradrenaline vs. terlipressin in the treatment of hepatorenal syndrome: a randomized study. J Hepatol. 2012;56:1293-8

30. Solanki P, Chawla A, Garg R, Gupta R, Jain M, Sarin SK. Beneficial effects of terlipressin in hepatorenal syndrome: a prospective, randomized placebo-controlled clinical trial. J Gastroenterol Hepatol. 2003;18:152-6.

31. Srivastava S, Shalimar, Vishnubhatla S, et al. Randomized controlled trial comparing the efficacy of terlipressin and albumin with a combination of concurrent dopamine, furosemide, and albumin in hepatorenal syndrome. J Clin Exp Hepatol. 2015;5:276-85.

32. Tavakkoli H, Yazdanpanah $\mathbf{K}$, Mansourian $\mathbf{M}$. Noradrenaline versus the combination of midodrine and octreotide in patients with hepatorenal syndrome: randomized clinical trial. Int J Prev Med. 2012;3:764-9.

33. Zafar S, Haque I, Tayyab GU, Khan G, Chaudry N. Role of terlipressin and albumin combination versus albumin alone in hepatorenal syndrome. Am J Gasteroenterol. 2012;107:S175-S176.

34. Briglia AE, Anania FA. Hepatorenal syndrome. Definition, pathophysiology, and intervention. Crit Care Clin. 2002;18:345-73.

35. Erly B, Carey WD, Kapoor B, McKinney JM, Tam M, Wang W. Hepatorenal syndrome: a review of pathophysiology and current treatment options. Semin Intervent Radiol. 2015;32:445-454.

36. Wadei HM, Mai ML, Ahsan N, Gonwa TA. Hepatorenal syndrome: pathophysiology and management. Clin J Am Soc Nephrol. 2006; 1: 1066-79.

37. Kiszka-Kanowitz M, Henriksen JH, Hansen EF, Moller S, Bendtsen F. Effect of terlipressin on blood volume distribution in patients with cirrhosis. Scand J Gastroenterol. 2004;39:486-492.

38. Nassar Junior AP, Farias AQ, D'Albuquerque LA, Carrilho FJ, Malbouisson LM. Terlipressin versus norepinephrine in the treatment of hepatorenal syndrome: a systematic review and meta-analysis. PLoS One. 2014;9:e107466

39. Ginès P. Hepatorenal syndrome, pharmacological therapy, and liver transplantation. Liver Transpl. 2011;17:1244-6. 